\title{
OFDM-based Massive MIMO Channel Estimation using Gaussian Mixture Learning and Compressed Sensing Methods
}

\author{
T. Ravi Babu, C. Dharma Raj, V. Adinarayana
}

\begin{abstract}
Massive MIMO-OFDM system is proved to be an effective and most sustainable technology to forthcoming applications of $5 G$ wireless communications. It furnished significant gains that facilitate a higher number of user connections at high data rates with improved latency and reliability. To achieve accurate channel knowledge, lessen pilot overhead is necessary. To resolve this problem, one of the favorite approaches is compressed sensing. Sparse channel estimation develops the essential sparsity between the communicating channels that can be improved by the channel estimation efficacy with lower pilot overhead. To achieve this, non-zero vector distribution can be taking into consideration the Gaussian mixture accordingly, learn their characteristics towards the expectation-maximization procedure. The results of simulation have proved the performance of proposed estimation approach of channel keeping with minimum pilot overhead and developed exceptional symbol error rate (SER) performance of the system.
\end{abstract}

Index Terms:Massive MIMO-OFDM,Gaussian Mixture, Approximate message passing, Channel estimation Compressed sensing.

\section{INTRODUCTION}

The Massive MIMO-OFDM is preeminent and supportive technology to $5 \mathrm{G}$ wireless applications that has to maintain excellent data rate and accuracy [1]. To achieve these eminent properties, knowledge of channel information is a most challenging issue in massive MIMO-OFDM systems, therefore, it is necessary to apply relevant estimation techniques to channels between all transmitting and receiving antennas accordingly. In this connection, by the use of proper training sequence design one can acquire accurate channel estimation [6,7]. However, with the help of least square (LS) or minimum mean square error (MMSE) methods to estimate the channel, they are non-supportive for adequate performance due to high computational complexity.

In general, communicating channels are sparse inherently; however, the majority of channels viewed as zero coefficients at channel impulse response (CIR). With a focus on channel sparsity, we implemented the compressed sensing-Aided (CS-Aided) method to characterize the channel properties of the massive MIMO-OFDM model [1]. The key advantage of the proposed approach needs fewer pilots than conventional methods. Many of the researches focused on different Greedy

Revised Manuscript Received on December 13, 2019.

T.Ravi Babu, Research Scholar, Department of ECE,GITAM Institute of Technology, (GIT) GITAM, Andhra Pradesh Visakhapatnam, India.

C.Dharma Raj, Professor, Department of ECE, GITAM Institute of Technology, (GIT) GITAM, Andhra Pradesh Visakhapatnam, India.

V. Adinarayana, Professor, Department of ECE, Avanthi Institute of Engineering and Technology (AIET), Andhra Pradesh Vizianagaram, India. algorithms and Bayesian compressed sensing (BCS) methods [16] which are used to determine the channel estimation onto the concentrated system model. Nonetheless, channel sparsity level is to point out as prior information at the receiving side.

The sparsity adaptive matching pursuit (SAMP) provides high performance at a wide range of practical applications without channel sparsity. However, there is inconsistency between convergence speed and recovery accuracy because SAMP has maintained a constant step size [2,3]. OFDM is one of the modulating systems that provide to mitigate interference and crosstalk resulting from the conversion of the serial data stream into parallel data stream at different frequencies. OFDM massive MIMO compressed sensing based channel estimation is set as sparse and dense vectors. These vectors are a combination of zero and nonzero vectors respectively. On the perfect sparse recovery, the sparse signal is reconstructed through the support of the LS technique [12].

The expectation-maximization (EM) steps are established to accomplish quantities of estimation following a Gaussian mixture model. Furthermore, the generalized approximate message passing (GAMP) which is an active algorithm in i.i.d distributed random signal [14] is exploiting to develop the expectation step and also mitigate computational complexity. The fast iterative truncation algorithm (FITRA) which is for sparse representation that was elaborated in [8], which found that it has a significant possible convergence rate, provided a regularization parameter to achieve the MUI cancellation and also standardize the functioning of the algorithm. In current work, we established and compared with a renewed compressed sensing aided over Gaussian mixture algorithms for downlink massive MIMO-OFDM systems with a reference of $\mathrm{ZF}$ pre-coding technique respectively. To estimate the error performance, the truncated and Bernoulli Gaussian mixture procedures are considered and entrusted to the unknown signal.

Simulated results found that the suggested algorithms provide a substantial improvement in terms of computational difficulties. The remainder part of this work is partitioned as per the following. Second Section discusses downlink and estimation models of noise. The third section covers training sequence design and the principle of estimation to existing technique, the fourth section discusses OMP and Bayesian approaches, fifth section practical issues and finally sixth section concludes. 


\section{OFDM-BASED MASSIVE MIMO}

Let the Base Station (BS) have $M$ transmitting probes and provide $K$ independently single element antennaesystem $(M \gg 1)$, and the total OFDM pilot tones $N$ that are assumed in figure 1 . Indeed, the set of accessible pilot tones are separated into data transmission and guard band. Therefore, every pilot tone of the corresponding $K \times 1$ signal vector of $x(n)$, that includes pilot symbols of $K$ users and of corresponding $n^{\text {th }}$ transmission symbol time with the data vector to satisfy $\mathbb{E}\left\{\|\mathrm{x}(n)\|_{2}^{2}\right\}=1$.In accordance with pilot tone is to be set as $x(n)=0_{K \times 1}$. Consequently, there is no way to exist the signal within the guard band. Now, take into consideration Zero-Force (ZF) pre-coding spatial signal approach for removal of multi-user interference (MUI) at BS because collaborative sensing across the users is much complicated.

The pre-coding sparse signal vector on the $n^{\text {th }}$ pilot tone can be expressed as

$$
\boldsymbol{s}(n)=\boldsymbol{P}(n) x(n)
$$

Where $\boldsymbol{s}(n) \in \mathbb{C}^{M \times 1}$ and $\boldsymbol{P}(n) \in \mathbb{C}^{M \times K}$ are precoded vector of $n^{\text {th }}$ sub-carrier of $M$ antennas and pre-coding channel matrix of $n^{\text {th }}$ OFDM pilot tone respectively. In view of the fact that $K \ll M$, the $\mathrm{ZF}$ pre-coding spatial channel vector matrix is expressed by

$$
\boldsymbol{P}_{n}^{Z F}=\boldsymbol{X}_{n}^{H}\left(\boldsymbol{X}_{n} \boldsymbol{X}_{n}^{H}\right)^{-1}
$$

here $\boldsymbol{P}_{n}^{Z F}$ provides the inverse and of pseudo-inverse of $\boldsymbol{P}(n)$ of $n^{\text {th }}$ pilot tone in MIMO channel vector matrix. Then after pre-coding, every pre-coded vector $\mathbf{h}(n)$ is rearranged by $M$ antennae to the downlink OFDM conversion such as

$$
\left[\begin{array}{lll}
\boldsymbol{a}_{1} & \ldots & \boldsymbol{a}_{M}
\end{array}\right]=\left[\begin{array}{lll}
\mathbf{h}_{1} & \ldots & \mathbf{h}_{M}
\end{array}\right]^{T}
$$

Here $\mathbf{h}_{m} \in \mathbb{C}^{N \times 1}$ denotes the frequency-domain samples at the $m^{t h}$ antennae. Signals of time-domain can be achieved by the result of inverse discrete Fourier transform (IDFT). Thereafter, overcome the inter symbol interference (ISI) by inserting cyclic prefix (CP) to the corresponding time-domain signals of respective antennas. Finally these time varying quantity signals are changed as analog signals for channel transmission.

After eliminating the CPs, the Discrete Fourier Transform (DFT) serves to achieve the frequency-domain signals. The transformed received vector of N-point DFT inclusive of $K$ user signals are represented by

$$
\mathbf{F}(n)=\frac{1}{\sqrt{N}} \exp ^{\left(-\frac{j 2 \pi \mathrm{n}}{N}\right)} \quad 0 \leq n \leq N-1
$$

The signal corresponds to $K$ user symbols expressed as

$$
\boldsymbol{y}(n)=\mathbf{X}(n) \mathbf{h}(n)+\mathbf{z}(n)
$$

Where $\mathbf{X}(n)$ and $\mathbf{h}(n)$ are the vectors for channel belongs to $\mathbb{C}^{N \times 1}$ and $\boldsymbol{z}(n)$ is the receiver noise corresponding to $n^{\text {th }}$ transmitted symbol.
The received signal vector appears error free MUI signal as $\boldsymbol{y}(n)=\mathbf{h}(n)+\boldsymbol{z}(n)$, while adding of equation (1),(2) and (4) which is to be involved ZF pre-coding scheme [17].

The transmitted pilots of $n^{\text {th }}$ antenna can be expressed as $\quad\left(\mathbf{X}_{P}=\mathbf{X}_{p_{1}}, \mathbf{X}_{p_{2}}, \ldots, \mathbf{X}_{p_{N_{P}}}\right)^{T} \in \mathbb{C}^{N_{P} \times 1} \quad$, where $\left[p_{1}, p_{2}, \ldots, p_{N_{P}}\right]$ representation of a pilot location. The signal received at the $n^{\text {th }}$ antenna can be denoted as

$$
\boldsymbol{y}(n)=\sum_{i=1}^{L} \operatorname{diag} \mathbf{F}(n) \boldsymbol{s}(n)+\boldsymbol{z}(n)
$$

In this system model we consider 256 Base station antennas operates with 128 antenna users and 16-QAM constellation is used with 32 OFDM pilot tones. Due to hundreds of transmit antennas at BS, explicitly degrades their channels performance, and also causes high pilot overhead to estimatethe channels. Accordingly, it is necessary to mitigate the high pilot overhead in employing system towards higher datarates.

\section{CS-BASED CHANNEL ESTIMATION}

Consider generated DFT signal (Frequency domain) matrix and additive noise of massive MIMO-OFDM model of $n^{\text {th }}$ antenna that is denoted as

$$
\boldsymbol{y}_{P}=\sum_{i=1}^{L} \operatorname{diag}\left(\mathbf{X}_{P}\right) \mathbf{F}_{P} \mathbf{h}_{n}+\mathbf{z}_{P}
$$

To estimate unknown vector $\mathbf{h}_{n}$ of massive MIMO-OFDM system, the proposed CS recovery algorithm is employed. For channel estimation in OFDM, the Greedy iteration reconstruction schemes [4, 7-9] have delivered potential performance.

Nonetheless, in many conventional greedy algorithms requires priori information at receiver side. Moreover, the greedy iteration schemes suffer with inaccuracy and more computational complexity $[12,15]$. Either of these two schemes has been successfully detected the fading channel information [14]. However, the proposed CS recovery algorithm need not consider the channel level as a prior information. This iteration is in accordance with the Partial common support information (PCSI) and depending on an iteration threshold. The PCSI of the $n^{\text {th }}$ receive antenna is expressed by

$$
\mathbf{I}_{n}=\sum_{n=1}^{L} \mathbf{I}_{n}+(n-1) L
$$

In the CS based channel estimation algorithm the following notations are involved. Updated measurement matrix, Observation vector, column index, iteration threshold, updated index, estimated channel vector, and residual are expressed by $\mathrm{A}_{t}, Y_{P}, \lambda_{t}, \varepsilon, \Lambda_{t}, \mathrm{r}_{t}, \hat{\mathbf{h}}_{n, t}$ respectively [5]. 
There are two major challenges with applying OMP to massive data $[13,15]$. Firstly, the computation complexity and an iteration storage cost are relatively large and secondly, the single coefficient selection simultaneously requires the corresponding $k$ iterationsto estimatewith $k$ coefficient of $\boldsymbol{q}$. whenever, the $k$ iterations are increases that leads impracticablyslow down its performance. To estimate the channel to the CS-based massive MIMO-OFDM, the following algorithm used as given below.

Algorithm I:

CS-Based Channel Estimation of Massive MIMO-OFDM

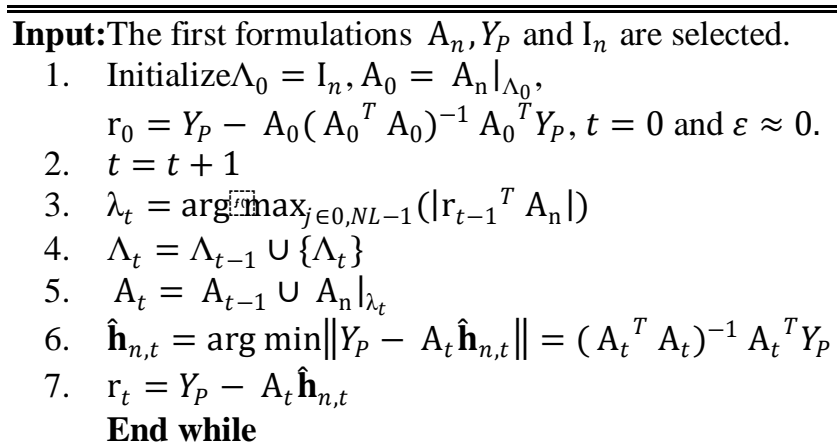

Output: estimate the CIR $\hat{\mathbf{h}}_{n, t}$.

CS based schemes (Static or Dynamic LS, OMP,CS-Aidedand BCS), the number of iterations are based on sparsity level of the channel $[3,6,11]$ whereas the proposed methodcloses iteration only when the residual is on the threshold of 0 . Consequently, the recovery accuracy can be assured. Moreover, when acquired accurate partial common support information, the number of iterations are limited; leads decrease incomputationalcomplexity of the proposed method.

To achieve this objective, we propose a Gaussianmixture (GM) methodical approachafterwardsby utilizing the expectation-maximization (EM) method [14] for determination of noisevariance and GM parameters. However, no need for concern quantitiesfor theEM updates because these computations made by suggested GAMP algorithm to decrease computational complexity [9]. The proposed generalized EM-TGM-AMP and EM-BGM-AMP can be effectively addressing at parametric estimation with i.i.d zero-mean Gaussian.

\section{GAUSSIAN MIXTURE GENERALIZED AMP}

To address the random Gaussian noise, generalizedAMP (GAMP) algorithm, proposal was made by Rangan [7, 16]. This proposed approach need not require knowledge about $p_{X}($.$) and the postulated noise variance, nevertheless it$ presents great recovery performance, that need to know about these postulatedinformation. In this Gaussian-mixture GAMP algorithm, we consider the coefficient in $\boldsymbol{x}=$ $\left[x_{1}, x_{2}, \ldots, x_{N}\right]^{T}$ isapproaching to thei.i.d distribution with marginalprobability density function can be expressed as

$$
\begin{aligned}
p_{X}(x ; \lambda, \boldsymbol{\omega}, \boldsymbol{\theta}, \phi) & =(1-\lambda) \delta(x) \\
& +\lambda \sum_{l=1}^{L} \omega_{l} \mathcal{N}\left(x ; \theta_{i}, \phi_{i}\right)
\end{aligned}
$$

Where $\delta(\cdot)$ function denotes Dirac delta identity and $\lambda$ is the percentageof sparsity rate. And $(\omega, \theta, \phi)_{k}$ coefficients are expressed as the weight, mean, and variance of $k^{\text {th }}$ GaussianMixture components respectively. In the remainder part $\sum_{i=1}^{L} \omega_{i}=1$ and zero mean and variance $\psi$ is used as noise. Here, the postulated parameters of GM-GAMP $\boldsymbol{q} \triangleq(\lambda, \boldsymbol{\omega}, \boldsymbol{\theta}, \boldsymbol{\phi}, \psi)$ are taken into consideration as known and fixed variables. Firstly, carry out the conditional distribution $p_{Y \mid Z}\left(y_{n} \mid z_{n} ; \boldsymbol{q}\right)$; the expanded postulated approximation can be expressed as

$$
p_{Z \mid Y}\left(z_{n} \mid \boldsymbol{y} ; \hat{p}_{n}, \mu_{n}^{p}, \boldsymbol{q}\right) \triangleq \frac{p_{Y \mid Z}\left(y_{n} \mid z_{n} ; \boldsymbol{q}\right) \mathcal{N}\left(z_{n} ; \hat{p}_{n}, \mu_{n}^{p}\right)}{\int_{z} p_{Y \mid Z}\left(y_{n} \mid z ; \boldsymbol{q}\right) \mathcal{N}\left(z ; \hat{p}_{n}, \mu_{n}^{p}\right)}(10)
$$

The moments of above density function under AWGN assumption isas

$$
\begin{aligned}
& E_{Z \mid Y}\left\{z_{n} \mid \boldsymbol{y} ; \hat{p}_{n}, \mu_{n}^{p}, \boldsymbol{q}\right\}=\hat{p}_{n}+\frac{\mu_{n}^{p}}{\mu_{n}^{p}+\psi}\left(y_{n}-\hat{p}_{n}\right) \\
& \left.\operatorname{var}_{Z \mid Y}\left\{z_{n} \mid \boldsymbol{y} ; \hat{p}_{n}, \mu_{n}^{p}, \boldsymbol{q}\right\}=\frac{\mu_{n}^{p} \psi}{\mu_{n}^{p}+\psi}\right)
\end{aligned}
$$

Secondly for computing the conditional distribution $p_{Y \mid Z}\left(x_{i} \mid \boldsymbol{y} ; \boldsymbol{q}\right)$; the expanded postulated approximation can be expressed as

$$
p_{X \mid Y}\left(x_{i} \mid \boldsymbol{y} ; \hat{r}_{i}, \mu_{i}^{r}, \boldsymbol{q}\right) \triangleq \frac{p_{X}\left(x_{i} ; \boldsymbol{q}\right) \mathcal{N}\left(x_{i}, \hat{r}_{i}, \mu_{i}^{r}\right)}{\int_{X} p_{X}(x ; \boldsymbol{q}) \mathcal{N}\left(x, \hat{r}_{i}, \mu_{i}^{r}\right)}
$$

To achieve subsequent approximation of GM-GAMP substitute the postulated parameters (3) into (8) and simplified expression can be given as

$$
\begin{aligned}
& p_{X \mid \boldsymbol{Y}}\left(x_{i} \mid \boldsymbol{y} ; \hat{r}_{i}, \mu_{i}^{r}, \boldsymbol{q}\right)= \\
& {\left[(1-\lambda) \delta\left(x_{i}\right)+\lambda \sum_{l=1}^{L} \omega_{l} \mathcal{N}\left(x_{i} ; \hat{r}_{i}, \mu_{i}^{r}\right)\right] \frac{\mathcal{N}\left(x_{i} ; \hat{r}_{i}, \mu_{i}^{r}\right)}{\varsigma_{i}}} \\
& \quad=\left[\left(1-\pi_{i}\right) \delta\left(x_{i}\right)+\pi_{i} \sum_{l=1}^{L} \bar{\beta}_{i, l} \mathcal{N}\left(x_{i} ; \gamma_{i, l}, v_{i, l}\right)\right]
\end{aligned}
$$

In (13) $\pi_{i}$ indicates posterior support probability values, $\operatorname{Pr}\left\{x_{i} \neq 0 \mid \boldsymbol{y} ; \boldsymbol{q}\right\}$ of GM-GAMPapproximation.

The normalized factor in (13) can be written as

$$
\begin{array}{r}
\varsigma_{i} \triangleq \int_{X} p_{X}(x ; \boldsymbol{q}) \mathcal{N}\left(x, \hat{r}_{i}, \mu_{i}^{r}\right)=(1-\lambda) \mathcal{N}\left(0 ; \hat{r}_{i}, \mu_{i}^{r}\right) \\
+\lambda \sum_{l=1}^{L} \omega_{l} \mathcal{N}\left(0 ; \hat{r}_{i}-\theta_{l}, \mu_{i}^{r}+\phi_{l}\right)
\end{array}
$$

Both (13) and (14) can be derived from (13a) through Gaussian probability density function multiplication rule.In (14) the following dependent variables can be given by

$$
\pi_{i} \triangleq \frac{1}{1+\left(\frac{\sum_{l=1}^{L} \beta_{i, l}}{(1-\lambda) \mathcal{N}\left(0 ; \hat{r}_{i}, \mu_{i}^{r}\right)}\right)^{-1}}
$$




$$
\begin{gathered}
\gamma_{i, l} \triangleq \frac{\frac{\hat{r}_{i}}{\mu_{i}^{r}}+\theta_{l} / \phi_{l}}{\frac{1}{\mu_{i}^{r}}+1 / \phi_{l}} \text { and } v_{i, l} \triangleq \frac{1}{\frac{1}{\mu_{i}^{r}}+1 / \phi_{l}} \\
\beta_{i, l} \triangleq \lambda \omega_{l} \mathcal{N}\left(0 ; \hat{r}_{i} ; \theta_{l}, \mu_{i}^{r}+\phi_{l}\right) \text { and } \bar{\beta}_{i, l} \triangleq \frac{\beta_{i, l}}{\sum_{l=1}^{L} \beta_{i, k}}
\end{gathered}
$$

To achieve effectiveness of GAMP approximation along with L-termGaussian Mixture is used to overcome difficulties onrealistic implementations. Using Bayesian parameter estimate method through AMPalgorithm [4, 26],provides accurate approximation that involvedwith central-limit-theorem, together with independent identicallydistributed zero-mean Gaussian $\boldsymbol{A}$.

\section{V.EM LEARNING OF THE PRIOR PARAMETERS}

Let us focus on learning of the priori parameters $\boldsymbol{q} \triangleq$ $(\lambda, \boldsymbol{\omega}, \boldsymbol{\theta}, \phi, \psi)$ with the help of expectation-maximization (EM) algorithm [5, 8,14]. The maximum likelihood $p(\boldsymbol{y} ; \boldsymbol{q})$ maximized a lower bound at the each iteration.The given illustration approximates the priori parameters by the following EM procedure.

$$
\begin{gathered}
\int_{x} \hat{p}(x) \ln p(\boldsymbol{y} ; \boldsymbol{q})=\int_{x} \hat{p}(x) \ln \left(\frac{p(\boldsymbol{x}, \boldsymbol{y} ; \boldsymbol{q})}{\hat{p}(x)} \frac{\hat{p}(x)}{p(\boldsymbol{x} \mid \boldsymbol{y} ; \boldsymbol{q})}\right) \\
E_{\hat{p}(x)}\{\ln p(\boldsymbol{x}, \boldsymbol{y} ; \boldsymbol{q})\}+H(\hat{p})+D(\hat{p} \| p \boldsymbol{x} \mid \boldsymbol{y}(\cdot \mid \boldsymbol{y} ; \boldsymbol{q}))
\end{gathered}
$$

Here $E_{\hat{p}(x)}\{\cdot\}, H(\hat{p})$ and $D(\hat{p} \| p)$ represents the expectation, entropy and Kullback-Leibler (K-L) divergence respectively. Here EM bounds are fixed as $\left(\boldsymbol{q}=\boldsymbol{q}^{n}\right)$ and $\left(\hat{p}=\hat{p}^{n}\right)$ respectively: expectation $\ln p\left(\boldsymbol{y} ; \boldsymbol{q}^{n}\right)-D\left(\hat{p} \| p_{\boldsymbol{X} \mid Y}\left(\cdot \mid \boldsymbol{y} ; \boldsymbol{q}^{n}\right)\right.$, afterwards the maximized function described as $\hat{p}^{n}(\boldsymbol{x})=$ $p_{\boldsymbol{X} \mid \boldsymbol{Y}}\left(\boldsymbol{x} \mid \boldsymbol{y} ; \boldsymbol{q}^{n}\right)$, maximization $E_{\hat{p}(x)}\{\ln p(\boldsymbol{x}, \boldsymbol{y} ; \boldsymbol{q})\}+H\left(\hat{p}^{n}\right)$, after that the maximized function produces as $\boldsymbol{q}^{n+1}=$ $\arg \max _{q} \widehat{\mathrm{E}}\left\{\ln p(\boldsymbol{x}, \boldsymbol{y} ; \boldsymbol{q}) \mid \boldsymbol{y} ; \boldsymbol{q}^{n}\right\}$ [6]. Where $\widehat{\mathrm{E}}$ implies the use of said posterior approximation.In addition to update the noise variance $\psi$ from determination of $\boldsymbol{q}^{n}$, therefore it can be expressed as

$$
\begin{array}{r}
\psi^{n+1}=\arg \max _{\psi>0} \sum_{m=1}^{M} \int_{x} p_{Z \mid \boldsymbol{Y}}\left(z_{m} \mid \boldsymbol{y} ; \boldsymbol{q}^{n}\right) \\
. \ln p_{\boldsymbol{Y} \mid Z}\left(y_{m} \mid z_{m} ; \psi\right)
\end{array}
$$

The maximized parameter $\psi$ that the derivative of the sum value is zero, in such way that can be obtain following noise variance parameter is,

$$
\psi^{n+1}=\frac{1}{M} \sum_{m=1}^{M}\left(\left|y_{m}-\hat{z}_{m}\right|^{2}+\mu_{m}^{z}\right)
$$

\section{A. EM Updates of BGM case}

Consider the marginal pdf of Bernoulli-Gaussian with $\ell_{1}$ GM model makes it possible to reduce to (17) then results as $p_{X}(x ; \lambda, \boldsymbol{\omega}, \boldsymbol{\theta}, \phi)=(1-\lambda) \delta(x)+\lambda \mathcal{N}(x ; \theta, \phi)$. Note that in this case need not require to find out the weight because its unity, so the prior benchmarks can be expressed by $\boldsymbol{q}^{n} \triangleq$ $\left(\lambda^{n}, \theta^{n}, \phi^{n}, \psi^{n}\right)$.

$$
\left.\lambda^{n+1}=\arg \max _{\lambda \in(0,1)} \sum_{i=1}^{L} \widehat{\mathrm{E}}\left\{\ln p_{X}\left(x_{i} ; \lambda, \boldsymbol{q}^{n}\right) \mid \boldsymbol{y} ; \boldsymbol{q}^{n}\right)\right\}
$$

The maximized parameter $\psi$ that the derivative of the sum value is zero, in such way that can be obtain following parameter is,

$$
\lambda^{n+1}=\frac{1}{L} \sum_{i=1}^{L} \pi_{i}
$$

In the similar way of (19), updatedEM parameters of $\theta$, $\phi$ are represented by

$$
\begin{gathered}
\theta^{n+1}=\frac{1}{\lambda^{n+1} N} \sum_{i=1}^{L} \pi_{i} \gamma_{i, 1} \\
\phi^{n+1}=\frac{1}{\lambda^{n+1} N} \sum_{i=1}^{L} \pi_{i}\left(\left|\theta^{n}-\gamma_{i, 1}\right|^{2}+v_{i, 1}\right)
\end{gathered}
$$

To estimate the channel performance to the CS-based massive MIMO-OFDM, the EM-BGM-AMP algorithm is used as given below.

\section{Algorithm II: EM-BGM-AMP}

$\overline{\text { Initialize the value of } L, \hat{x}^{0}=0 \text { and unknown parameter }}$ of $\boldsymbol{q}^{0}$.

For $n=1$ to $N_{\max }$ do

Generate $\hat{x}^{n}, \hat{z}^{n},\left(\mu^{z}\right)^{n}, \pi^{n},\left\{\beta^{n, k}, \gamma_{k}^{n}, v_{k}^{n}\right\}_{k=1}^{M}$ through BGM-GEMP with $\boldsymbol{q}^{n-1}$.

if $\left\|\hat{x}^{n}-\hat{x}^{n-1}\right\|_{2}^{2}<\tau_{E M}\left\|\hat{x}^{n-1}\right\|_{2}^{2}$ then

Break

end if

Compute $\lambda^{n}$ from $\pi^{n-1}$.

For $k=1$ to $M$ do

if sparse mode value enabled then

Compute $\theta_{k}^{n}$ from $\pi^{n-1}, \gamma_{k}^{n-1},\left\{\beta_{l}^{n-1}\right\}_{l=1}^{M}$.

end if

Compute $\phi_{k}^{n}$ from $\pi^{n-1}, \theta_{k}^{n-1}, \gamma_{k}^{n-1},\left\{\beta_{l}^{n-1}\right\}_{l=1}^{M}$.

end

Compute $\boldsymbol{\omega}^{n}$ from $\pi^{n-1}$ and $\left\{\beta_{l}^{n-1}\right\}_{l=1}^{M}$.

end

Compute $\psi^{n}$ from $\widehat{z}^{n}$ and $\left(\mu^{z}\right)^{n}$.

Apply the Leibniz's integral principleto interexchange of differentiate and integrate signs, the Dirac approximation using this $\delta(x)=\mathcal{N}(x ; 0, \varepsilon)$ for establishrandomly $\varepsilon>0$, thenit complete, and its differential coefficient with respect to $\lambda$ make steadily. The similar interpretationcan be addresses to all interexchange ofdifferentiation and integration in the sequence, also mentioned in section III.

\section{B. EM Updates of GM case}

Firstly we initiate EM update for $\lambda$ from priori parameters $\boldsymbol{q}^{n} \triangleq\left(\lambda^{n}, \boldsymbol{\omega}^{n}, \theta^{n}, \phi^{n}, \psi^{n}\right)$. Since $\lambda^{n}$ is presented in (19) BG case i.e. $\lambda^{n+1}=\frac{1}{L} \sum_{i=1}^{L} \pi_{i}$, so for concise not be repeated here. Now we consider the remaining parameters $\boldsymbol{\omega}, \boldsymbol{\theta}$ and $\phi$. In this case, $k=1,2, \ldots, L$, the

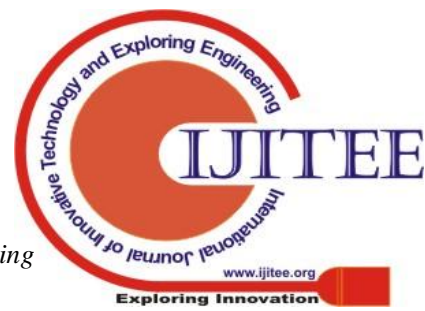


updates are incremented one by one that is $\theta_{k} \rightarrow \phi_{k} \rightarrow \boldsymbol{\omega}$,all these parameters are fixed variables. The Prior parameters in the form of $p_{X}(x)=(1-\lambda) \delta(x)+\lambda f_{X}$, is to provide randomly by $f_{X}(\cdot)$, the EMupdate for $\lambda$ is specified by (20). Therefore the EM updates are identified by

$$
\begin{aligned}
\theta_{k}^{n+1} & \left.=\arg \max _{\theta_{k} \in \mathbb{R}} \sum_{i=1}^{L} \widehat{\mathrm{E}}\left\{\ln p_{X}\left(x_{i} ; \theta_{k}, \boldsymbol{q}^{n}\right) \mid \boldsymbol{y} ; \boldsymbol{q}^{n}\right)\right\} \\
\phi_{k}^{n+1} & \left.=\arg \max _{\phi_{k}>0} \sum_{i=1}^{L} \widehat{\mathrm{E}}\left\{\ln p_{X}\left(x_{i} ; \phi_{k}, \boldsymbol{q}^{n}\right) \mid \boldsymbol{y} ; \boldsymbol{q}^{n}\right)\right\} \\
\boldsymbol{\omega}^{n+1} & \left.=\arg \max _{\theta_{k} \in \mathbb{R}} \sum_{i=1}^{L} \widehat{\mathrm{E}}\left\{\ln p_{X}\left(x_{i} ; \boldsymbol{\omega}, \boldsymbol{q}^{n}\right) \mid \boldsymbol{y} ; \boldsymbol{q}^{n}\right)\right\}
\end{aligned}
$$

The maximized value of $\theta_{k}$ is fundamentally requires zeros the derivative, i.e., that accomplished expression is

$$
\left.\sum_{i=1}^{L} \int_{x} p_{X \mid Y}\left(x_{i} \mid \boldsymbol{y} ; \boldsymbol{q}^{n}\right) \frac{d}{d \theta}\left\{\ln p_{X}\left(x_{i} ; \theta, \boldsymbol{q}^{n}\right) \mid \boldsymbol{y} ; \boldsymbol{q}^{n}\right)\right\}
$$

Substitute the maximized value (23) in (22a) then produces

$$
\sum_{i=1}^{L} \int_{x} p_{X \mid Y}\left(x_{i} \mid \boldsymbol{y} ; \boldsymbol{q}^{n}\right) \frac{d}{d \theta_{k}}\left\{\ln p_{X}\left(x_{i} ; \theta_{k}, \boldsymbol{q}^{n}\right) \mid \boldsymbol{y} ; \boldsymbol{q}^{n}\right\}=0
$$

Plugging in the derivative and apply the approximation $\mathcal{N}\left(x_{i} ; \theta_{k}, \phi_{k}^{n}\right) \approx \mathcal{N}\left(x_{i} ; \theta_{k}^{n}, \phi_{k}^{n}\right) \quad$ to numerator and denominator. Finally we acquired the simplified approximation coefficients are

$$
\theta_{k}^{n+1}=\frac{\sum_{i=1}^{L} \pi_{i} \beta_{i, k} \gamma_{i, k}}{\sum_{i=1}^{L} \pi_{i} \beta_{i, k}}
$$

It is found that the above EM approach gives a way to perfect achievement of MSE estimation at sparse signals [10]. In similar fashion the respective approximation parameters are also found to $\phi_{k}^{n+1}$ and $\omega_{k}^{n+1}$ that are

$$
\begin{gathered}
\phi_{k}^{n+1}=\frac{\sum_{i=1}^{L} \pi_{i} \bar{\beta}_{i, k}\left(\left|\phi_{k}^{n}-\gamma_{i, k}\right|^{2}+v_{i, k}\right)}{\sum_{i=1}^{L} \pi_{i} \bar{\beta}_{i, k}} \\
\omega_{k}^{n+1}=\frac{\sum_{i=1}^{L} \pi_{i} \bar{\beta}_{i, k}}{\sum_{i=1}^{L} \pi_{i}} .
\end{gathered}
$$

\section{C.EM Updates of TGM Case}

The advantage of GAMP method is to provide the approximation of the likelihood function $p(\boldsymbol{y} ; \boldsymbol{q})$ through $\hat{p}^{n}(\boldsymbol{x})=p_{\boldsymbol{X} \mid \boldsymbol{Y}}\left(\boldsymbol{x} \mid \boldsymbol{y} ; \boldsymbol{q}^{n}\right)$ and the EM produces a new estimate of $\boldsymbol{q}(x)$ and $\psi$, in addition to posteriors approximation of the other involved variables and determinate the boundary parameter $v$. The algorithm summarizes the proposed approach as follows.

For the concise of this work we do not repeat the posterior approximations here; new updates of noise variance present.

$$
\psi^{n+1}=\sum_{m=1}^{M} p_{Z \mid \boldsymbol{Y}}\left(z_{m} \mid \boldsymbol{y} ; \boldsymbol{q}^{n}\right) \ln p_{\boldsymbol{Y} \mid Z}\left(y_{m} \mid z_{m} ; \psi\right)+\mathrm{const}
$$

$$
=\frac{N}{2} \ln \psi-\frac{1}{2} \psi \sum_{m=1}^{M}\left(\left|y_{m}-\hat{z}_{m}\right|^{2}+\mu_{m}^{z}\right)
$$

The new approximate parameter of $\psi$ is can be solved by setting to fundamental requirement of zeros the derivative refer (24), that leads an achieved expression is

$$
\psi^{n+1}=\frac{M}{\sum_{m=1}^{M}\left(\left|y_{m}-\hat{z}_{m}\right|^{2}\right)}
$$

To estimate the channel performance to the CS-based massive MIMO-OFDM, the EM-TGM-AMP algorithm is used as given below.

\section{Algorithm II: EM-TGM-AMP}

The first formulations $\psi^{(0)}, v^{(0)}$ are selected.

1. Initialize the mean, variance parameters of $\boldsymbol{q}(x)$ and

GAMP iterations the iteration number, $t$, with zero.

Repeat untilt $\geq t_{\max }$

2. Calculate the approximate distribution $\hat{p}(\boldsymbol{x} \mid \boldsymbol{y} ; \boldsymbol{q})$ and

$$
\hat{p}_{Z \mid \boldsymbol{Y}}\left(z_{m} \mid \boldsymbol{y} ; \boldsymbol{q}^{n}\right)
$$

3. Utilize the approximate likelihoods $\hat{p}(\boldsymbol{x} \mid \boldsymbol{y} ; \boldsymbol{q})$, update Posterior variables $\widehat{\boldsymbol{q}}^{n}\left(\lambda^{n}, \boldsymbol{\omega}^{n}, \theta^{n}, \phi^{n}\right)$

4. Compute noise variance $\widehat{\psi}^{n+1}$ from and obtain $\hat{v}^{n+1}$ 5. $t=t+1$.

Return to step 2.

Now consider the boundary parameter $v$, to achieve this choose the mean of posterior distributed parameter of $\boldsymbol{q}(x)$.

$$
v^{n+1}=v^{n}+\Delta_{v}
$$

Where $\Delta_{v}$ step-size of boundary points is: expand the boundary point $v$ for that adequately smaller step-size $\Delta_{v}$. Here we may be able to anticipate towards the signal $x$ will be increase as a result.

\section{SIMULATION RESULTS AND DISCUSSIONS}

The efficiency of proposed truncated Gaussian mixture EM-GAMP and Gaussian Bernoulli EM-GAMP algorithm can be compared in accordance with obtained results of zero-forcing $(\mathrm{ZF})$ pre-coding OMP andCS-Aided approach.

Fig. 1 has presented the comparison of various schemes of NMSE performance withknownerror value of $k_{s}^{e}$ (assume estimate channel coefficient $k_{s}=2$ ). The estimation methods are employing with the previous support informationshows the poor performance upon theunbalanced parameter arises, whereas the quality of support information is trivial to the proposed approaches. Because the imperfect channelcoefficients are evaluatedby a compressed sensing (CS) algorithm and has low mismatched influence. In addition, iferroneouscoefficients are selectedby this method that clipping step can terminate the impact of erroneous coefficients. In addition, proposed and CS-Aided methods can be maintained the constant support information. 
Fig. 2 indicates the performance of NMSE versus SNR with various schemes with varying SNR depends on the pilot tone performance. Since, by considering noise level CS algorithms are fundamentally sensitive, the estimation methods are employing with $\mathrm{CS}$, including the considered approaches, yields great outcome in range of SNR.Moreover, the CS-Aided, OMP and ZF pre-coding scheme show comparatively low error. And the SNR between $13 \mathrm{~dB}$ to 15 $\mathrm{dB}$, the proposed schemes produces better results than $\mathrm{ZF}$ and OMP. Throughout the simulation results on figure 3 , the proposed schemes can be obtained the lowest error rate than $\mathrm{ZF}$ and the basic greedy algorithm is called the OMP.

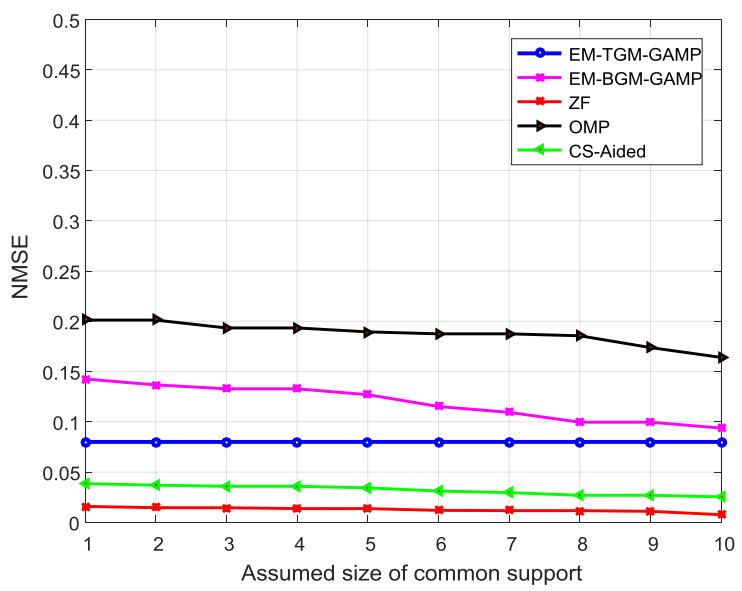

Fig.1. NMSE vs mismatched parameter with $M=128$, $k=$ 64, and $\mathrm{SNR}=20 \mathrm{~dB}$.

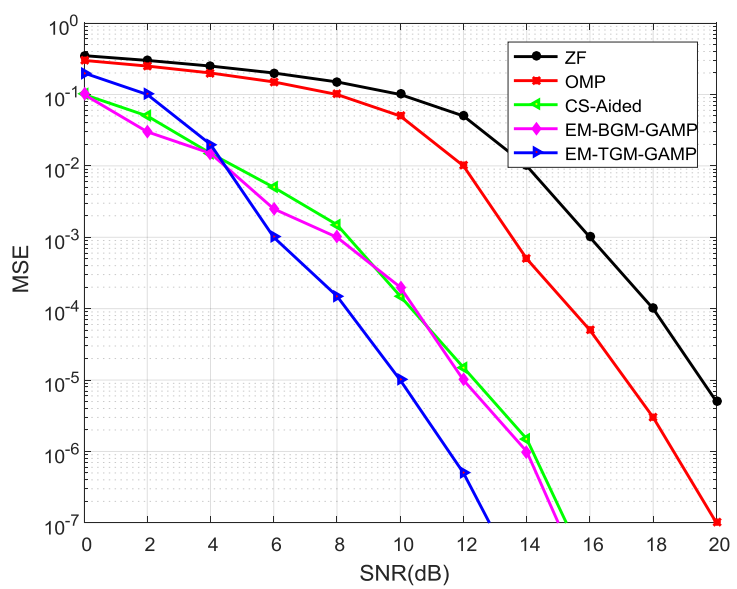

Fig. 2. MSE versus SNR recovery performance of various schemes

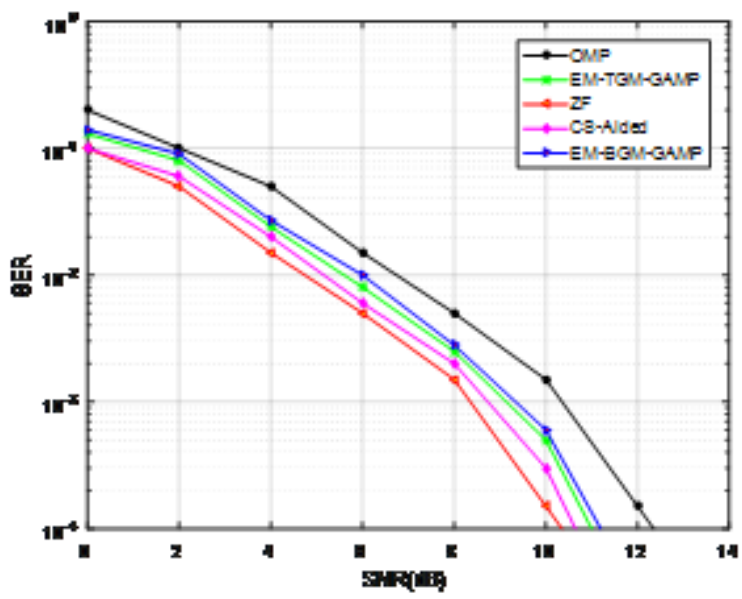

Fig. 3. SER versus SNR recovery performance of various schemes
The effectiveness MSE with different algorithms is proved in Fig.3. It appears that the suggested schemes are achievedbetter Signal to noise ratio (SNR) performance as against the ZF pre-coding. However, the ZF provides the least-norm solution.

In order to sustain the same, must have to remove the undesirable characteristics, i.e. perfect normalization is must. In comparison to this, LS based schemes have less error. For SNR between 10-11 dBs, the proposed schemes are better than $\mathrm{ZF}$ and as reasonably good performance. Overall, the performed proposed methods have less error than conventional CS techniques.

According to estimate made by comparison, the GM-based CS approaches are easy to determine better training signals. Thus, quality of channel estimate is evaluated by the intended GM-based CS approaches which provides better performance through flagship challenge ofthe trainingsignals.

\section{CONCLUSION}

This work focused on estimate the channel efficacy over OFDM based massive MIMO downlink system under Gaussian mixture learning with various approaches for compressed sensing. Particularly the composition of the GAMP technique with the EM iterative methods and they facilitate the less computational complexity through designed pilot approach. Through the continuous support of the CS-Aided approach, the pilot overhead is reduced. The favorable channel performance is achieved by the use of EM-TGM-GAMP, EM-BGM-GAMP, and CS-Aided schemes. The obtained simulation figures give the truncated GM appears better performance than GB distribution in $\mathrm{CS}$, and also achieved better output compared to the ZF and OMP techniques.

\section{REFERENCES}

1. E. G. Larsson, F. Tufvesson, et.all, "Massive MIMO for next generation wireless systems," IEEE CommunicationMagazine. volume. 52, no. 2, pp. 186-195, February. 2014.

2. U. S. Kamilov, S. Rangan, et.all, "Approximatemessage passing with consistent parameter estimation and applicationsto sparse learning," in Proceedings. Neural Inform. Process System, Conf., Dec. 2012

3. R. Neal and G. Hinton, "A view of the EM algorithm that justifiesincremental, sparse, and other variants," in Learning in GraphicalModels (M. I. Jordan, ed.), pp. 355-368, MIT Press, 1999.

4. Y.Han, J. Lee and David J.Love, "Compressed Sensing-Aided Downlink Channel Training for FDD Massive MIMO Systems" IEEE Transactions. On Communications, Volume: 65, Issue: 7, July 2017

5. J. Ziniel and P. Schniter, "Dynamic compressive sensing of time-varying signals via approximate message passing," IEEE Signal Process, volume 61, no. 21, pp. 5270-5284, November 2013.

6. C. R. Berger, Z. Wang, J. Huang, and S. Zhou, "Application of compressive sensing to sparse channel estimation," IEEE Commun. Magazine, volume 48, no 11, pp 164-174, November 2010.

7. S. Rangan, "Generalized approximate message passing for estimation with random linear mixing," in Proc. IEEE Int. Symp. Information Theory (ISIT), Saint Petersburg, Russia, Aug. 2011, pp. 2168-2172.

8. D. L. Donoho, A. Maleki, and A. Montanari, "Message passing algorithms for compressed sensing," Proc. Nat. Acad. Sci., vol. 106, no. 45, pp. 18 914-18 919, 2009.

9. M. Bayati and A. Montanari, "The dynamics of message passing on dense graphs, with applications to compressed sensing",IEEE Transactions. Inf. Theory, volume. 57, no. 2, pp. 764-785, Feb. 2011 
10. M. P. Friedlander, H. Mansour, et.al, "Recovering " compressively sampled signals using partial support information," IEEE Transactions Inf. Theory, volume 58, no. 2, pp. 1122-1134, Feb. 2012

11. W. U. Bajwa, J. Haupt, et.al, "Compressed channel sensing: A new approach to estimating sparse multipath channels," Proceedings. IEEE, volume 98, no 6, pp 1058-1076, June 2010.

12. N. Vaswani, "LS-CS-residual (LS-CS): Compressive sensing on least squares residual," IEEE Transactions, Signal Process, volume. 58, no. 8, pp. 4108-4120, August 2010.

13. Y. C. Pati, R. Rezaiifar, et.al, "Orthogonal matching pursuit: Recursive function approximation with applications to wavelet decomposition," in Proceedings. 27th Annual Asilomar Conference. Signals, Systems, and Computers, 1993, pp. 40-44.

14. P. Vila and P. Schniter, "Expectation-maximization Gaussian-mixture approximate message passing," IEEE Trans. Signal Processing., volume 61, no. 19, pp. 4658-4672, Oct. 2013.

15. R. Wu and D. Chen, "The exact support recovery of sparse signals with noise via orthogonal matching pursuit," IEEE Signal Process. Letters., volume.20, no. 4, pp. 403-406, April 2013.

16. S. Ji, Y. Xue, and L. Carin, "Bayesian compressive sensing," IEEE Transactions,Signal Processing., vol. 56, pp. 2346-2356, June 2008.

17. H. Q. Ngo, E. G. Larsson, and T. L. Marzetta, "Massive MU-MIMO downlink TDD systems with linear precoding and downlink pilots," in Allerton Conference, Communication, Control, and Computing, UrbanaChampaign, Illinois, October. 2013, pp. 293-298.

\section{Authors Profile}

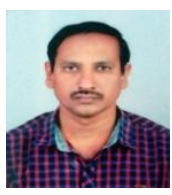

T. Ravi Babu received his B.Tech and M.Tech degrees in Electronics and Communication Engineering from JNTU University college of Engineering, Kakinada, India, in 2005 and 2010 respectively. He is a Research scholar in GITAM Institute of Technology (GIT), GITAM, Visakhapatnam.His area of research includesChannel estimation on $4 \mathrm{G} / 5 \mathrm{G}$ wireless communications, MIMO-OFDM, Massive MIMO, fading Channels. He has 10 years teaching 7 years Industrial experience. He has supervised and guided various projects for UG and PG level. He is a Member of Indian Society for Technical Education (MISTE).

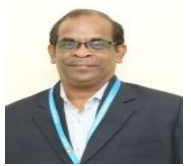

Prof. C. Dharma Raj is currently working as Professor in Dept. of E.C.E., GITAM Institute of Technology (GIT), GITAM, Visakhapatnam, Andhra Pradesh, India. He received his B.E. in Electronics \& Communication Engineering from Andhra University and M.E. in Microwave \& Radar Engineering from Osmania University in 1985 and 1987 respectively. He received his Ph. D in 2012 from GITAM. He worked as Scientist "B" in LRDE, Bangalore for about one year and later he worked in Marine and Communication Electronics India Ltd., as Technical officer for three years. Ever since, after his work in industry, he is working in GITAM and served many positions for the past 29 years. He had published 38 papers in Reputed Journals like Springer, IEEE etc., and he also authored 7 text books in the field of E.C.E and is guiding $7 \mathrm{PhD}$ students -2 of his $\mathrm{PhD}$ students wereawarded degrees. He has supervised $28 \mathrm{M}$. Tech projects. His area of research includes Electromagnetic field theory, Antennas, Microwave \& Radar Engineering and Wireless Communications.

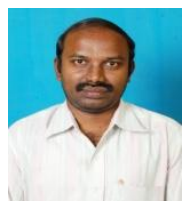

Dr. V. Adinarayana Received B.E. Degree in Electronics \& Communication Engineering from G.I.T.A.M Engineering College, Affiliated to Andhra University, Andhra Pradesh (A.P), India in 2000. Did his M.E in 2003 from Andhra University College of Engineering. He has secured national $8^{\text {th }}$ Rank in AURCET for Ph.D. entrance test, Awarded Ph.D. degree in Mobile \& Wireless Communications in 2019 from Faculty of Engineering, Dept. of ECE, Andhra University, India. Worked as Assistant professor at GMRIT Engg College, Andhra Pradesh (A.P), India, Associate professor\&HoD in SVP Engg College, Visakhapatnam, AP, India and as Associate Professor in VIIT, Vignan's Group, Duvvada, and Visakhapatnam. He has 16 years of teaching experience. At present working as Professor in ECE Department at Avanthi Institute of Engineering and Technology (AIET), cherukupally, Vizianagaram, AP, India, He has guided various research projects for UG, PG level students. Published research articles in Both National and International reputed journals and presented papers in IEEE international conferences at different locations. His interesting research Area is mobile and wireless communications. 